Original Article

\title{
Relationship between spinal range of motion and trunk muscle activity during trunk rotation
}

\author{
Tomoaki Sugaya, RPT, MS ${ }^{1,2)^{*}}$, Masaaki Sakamoto, RPT, PhD ${ }^{1)}$, Rie Nakazawa, RPT, PhD ${ }^{1)}$, \\ NAOKI WADA, MD, $\mathrm{PhD}^{3)}$ \\ 1) Graduate School of Health Sciences, Gunma University, Japan \\ 2) Department of Rehabilitation, Gunma University Hospital: 3-39-15 Syowa, Maebashi, Gunma 371- \\ 8511, Japan \\ 3) Department of Rehabilitation Medicine, Gunma University Graduate School of Medicine, Japan
}

\begin{abstract}
Purpose] The aim of this study was to clarify the relationship between spinal range of motion and trunk muscle activity during trunk rotation using a three-dimensional motion analysis system and surface electromyography. [Subjects and Methods] The subjects comprised 11 healthy men. A three-dimensional motion analysis system measured the trunk rotational angle of 4 segments of the thoracic vertebrae and 2 segments of the lumbar vertebrae. Surface electromyography measured the activities of the unilateral latissimus dorsi, lumbar multifidus, rectus abdominis, external oblique, internal oblique, and transversus abdominis muscles. [Results] During ipsilateral rotation at thoracic vertebral levels, the muscle activity of the latissimus dorsi and external oblique was significantly increased compared with the activity in the $0-10 \%$ range of trunk rotation. During early ipsilateral rotation at lumbar vertebral levels, the muscle activity of the internal oblique and transversus abdominis was significantly increased compared with that in the $0-10 \%$ range of trunk rotation. During contralateral rotation at both thoracic and lumbar vertebral levels, the muscle activity of the external oblique was significantly increased compared with that in the $0-10 \%$ range of trunk rotation. [Conclusion] This study indicates that it is important to consider vertebral segments and spinal range of motion during trunk rotation.

Key words: Trunk rotation, Three-dimensional motion analysis, Surface electromyography
\end{abstract}

(This article was submitted Oct. 8, 2015, and was accepted Nov. 17, 2015)

\section{INTRODUCTION}

Trunk rotation is involved in various activities, from walking to sports. Trunk rotation is a key risk factor for low back pain $^{1,2)}$. During rotation, the external oblique (EO), rectus abdominis (RA) and lumbar multifidus (MF) muscles act contralaterally, whereas the latissimus dorsi (LD), internal oblique (IO) and transversus abdominis (TrA) muscles act ipsilaterally ${ }^{3-6)}$. Trunk rotation is a motion involving both thoracic and lumbar vertebrae. However, previous studies have only examined trunk rotation in a general sense. Furthermore, little has been reported about the relationship between the level of trunk muscle activity and spinal range of motion during trunk rotation. Recently, a method for calculating the rotational angle of each spinal segment during trunk rotation was developed using a three-dimensional motion analysis in trunk rotation ${ }^{7)}$. Therefore, the range of motion during trunk rotation for specific segments of the spinal column was evaluated in the current study. The aim of this study was to clarify the relationship between spinal range of motion and trunk muscle activity during trunk rotation.

\footnotetext{
*Corresponding author. Tomoaki Sugaya (E-mail: t.sugaya@gunma-u.ac.jp)

(C)2016 The Society of Physical Therapy Science. Published by IPEC Inc.

This is an open-access article distributed under the terms of the Creative Commons Attribution Non-Commercial No Derivatives (by-nc-nd) License $<$ http://creativecommons.org/licenses/by-nc-nd/4.0/>.
} 


\section{SUBJECTS AND METHODS}

Subjects

The study subjects consisted of 11 healthy volunteer men with no history of orthopedic or neurological disorders and no lumbar symptoms during the prior 3 months (age: $26.5 \pm 3.3$ years; height: $173.2 \pm 4.5 \mathrm{~cm}$; weight: $65.4 \pm 3.9 \mathrm{~kg}$ ). The Ethics Committee of Gunma University approved this study (approval code 15-30) which was conducted in accordance with the Declaration of Helsinki. Written informed consent was obtained from all participants and the rights of all subjects were protected.

\section{Methods}

Surface electromyography (EMG) was acquired using a cordless EMG (MQAir, Kissei Comtec Co., Ltd., Japan) with a $10-1,000 \mathrm{~Hz}$ bandwidth and a common mode rejection ratio $>93 \mathrm{~dB}$. Muscle activity was recorded over the two right back muscles and the four abdominal muscles: LD, MF, RA, EO, IO and TrA. Electrode placement is shown in Table $1^{8-12)}$. In our previous study, it was reported that the IO and TrA performed the same functions ${ }^{13)}$; therefore, it was the same electrode placement of the IO and TrA in this study. All recordings were acquired using hydro-gel and disposable $\mathrm{Ag} / \mathrm{AgCl}$ electrodes $(38 \mathrm{~mm} \times 19 \mathrm{~mm})$. Following careful skin preparation, electrodes were placed parallel to the muscle fibers with a center-tocenter distance of $30 \mathrm{~mm}$. As a normalization reference, EMG data were sampled during three 5-s trials of manually resisted maximal voluntary contraction (MVC) for each muscle.

Marker positions were acquired using a motion capture system (VICON Motion System Ltd., Oxford, UK), operating at a sampling rate of $200 \mathrm{~Hz}$. A total of 22 reflective markers, each with a 9.5-mm diameter, were placed on each of the seven spinal segments, according to the method described by Preuss et $\mathrm{al}^{7}$ ). The sacral segment ( $\mathrm{Sx}$ ) was defined by three markers over the posterior superior iliac spines and the fifth sacral vertebra (S5). Similarly, the lower lumbar segment (LLx) was defined by three markers placed $50 \mathrm{~mm}$ lateral to the spinous process of the third lumbar vertebra (L3) and over the first sacral vertebra (S1). Moving rostrally, each successive segment was defined by a centrally placed marker below, and two laterally placed markers above. These markers were placed over, and lateral to, the spinous processes of the L3, 12th, 9th, 6th and 3rd thoracic vertebrae (T12, T9, T6 and T3) and the 7th cervical vertebra (C7). The segments defined by these marker groups were termed upper lumbar (ULx), lower thoracic (LTx), mid-lower thoracic (MLTx), mid-upper thoracic (MUTx) and upper thoracic (UTx) segments, respectively.

Subjects sat with their feet flat on the ground and their arms crossed over their chests. The hip and knee joints were flexed at $90^{\circ}$ and the thighs were immobilized with a belt. Rotation began at the $-10^{\circ}$ position from the acromion level and proceeded in a positive direction until maximum rotation was achieved. Measurements were recorded for each direction. For all measurements, the trunk rotation position of the subjects was corrected. Movement progressed at a speed sufficient to complete the rotation in approximately $3 \mathrm{~s}$.

Using the analytical software KineAnalyzer (Kissei Comtec Co., Ltd., Japan), the raw EMG data and kinematics data were synchronized. All EMG signals were filtered using a 30-500 Hz band-pass filter and all EMG data were converted to root mean square (RMS) values. The RMS values of MVC were obtained by averaging over 3 trials, the mean value for $3 \mathrm{~s}$ of stable waveform in the middle of the recording to provide EMG signal amplitude normalization. For kinematic data, the normal vector of Sx was defined as the basic axis and the normal vector of the other segments was defined as the moving axis. Furthermore, the trunk rotational angle was defined as the angle of the horizontal components in the angle formed by the two normal vectors. The range of trunk rotation for each segment was calculated using the degree positions at the maximal trunk rotation and the $0^{\circ}$ point. For each segment, a normalized ratio of the range was obtained by calculating the ratio of the observed range to the range of maximal trunk rotation at the UTx. Using this result, each segment's maximal degree of rotation and the ratio at the UTx were calculated. In addition, the percentage of the MVC for each muscle split range at an interval of $10 \%$ of the ratio was calculated for each segment.

Statistical analyses were performed using IBM SPSS Statistics (Version 22) for Windows. Comparisons of the percentage of the MVC during the $0-10 \%$ range of trunk rotation and each split range at an interval of $10 \%$ were performed with a

Table 1. Electrode placement for EMG recording of the trunk musculature

\begin{tabular}{ll}
\hline Muscle & Surface electrode placement \\
\hline LD & lateral to 9 th thoracic vertebra over the muscle belly \\
MF & $3 \mathrm{~cm}$ lateral to 4th lumbar vertebra spinous process \\
RA & $1 \mathrm{~cm}$ above the umbilicus and $2 \mathrm{~cm}$ lateral to the midline \\
EO & lower edge of the eighth rib \\
IO/TrA & $2 \mathrm{~cm}$ below the line between the anterior superior iliac spines and medial to the inguinal ligament \\
\hline LD: latissimus dorsi, MF: lumbar multifidus, RA: rectus abdominis, EO: external oblique, IO/TrA: internal oblique \\
and transversus abdominis
\end{tabular}


Wilcoxon signed rank test with a significance level of 5\%. A Bonferroni adjustment with a significance level of $0.55 \%$ was performed for all significant differences.

\section{RESULTS}

Each segment's maximal degree of rotation and the ratio to that observed for the UTx are reported in Table 2.

As demonstrated in Table 3, during right (ipsilateral) rotation, the ratio for the LD and EO was significantly increased for all split ranges more than $40-50 \%$ in the UTx. Similarly, the ratio for the MF was significantly increased for all split ranges more than $80-90 \%$. In the MUTx, the ratio for the LD and EO was significantly increased for all split ranges more than $80-90 \%$. In the MLTx, the ratio for the MVC in the LD and MF was significantly increased for all split ranges more than 70-80\%; for the EO, all split ranges more than 60-70\%; and for the IO and TrA, predominantly in the 50-60\% split range. In the LTx, the ratio for the LD was significantly increased for all split ranges more than 50-60\%; for the MF, all split ranges more than 70-80\%; and for the EO, and all split ranges more than 40-50\%. In the ULx, the ratio for the LD was significantly increased for all split ranges more than 60-70\%; for the MF, all split ranges more than 80-90\%; for the EO, all split ranges more than 40-50\%; and for the IO and TrA, all split ranges more than 20-30\%. In the LLx, the ratio for the LD was significantly increased for all split ranges more than 80-90\%; for the MF, all split ranges more than 70-80\%, except for the $80-90 \%$ split range; for the EO, all split ranges more than 50-60\%; and for the IO and TrA, all split ranges more than 20-30\%, except for the 60-70\% split range.

As demonstrated in Table 4, during left (contralateral) trunk rotation, the ratio for the LD was significantly increased for all split ranges more than $80-90 \%$ in the UTx. Similarly, the ratio for the EO was significantly increased for all split ranges more than 30-40\%. In the MUTx and MLTx, the ratio for the EO was significantly increased for all split ranges more than 30-40\%. In the LTx, the ratio for the LD was significantly increased for all split ranges more than $70-80 \%$, except for the $80-90 \%$ split range; and for the EO, all split ranges more than $40-50 \%$. In the ULx, the ratio for the EO was significantly increased for all split ranges more than $40-50 \%$, except for the $60-70 \%$ split range. In the LLx, the ratio for the EO was significantly increased for all split ranges more than $40-50 \%$.

Under all conditions, there were no significant differences in the ratio for the RA.

\section{DISCUSSION}

In this study, trunk rotational angle was identical for the UTx and MUTx. Previous studies reported that the change in trunk rotational angle from $\mathrm{C} 7$ to $\mathrm{T} 3$ and from $\mathrm{T} 3$ to $\mathrm{T} 6$ were identical ${ }^{14,15}$. The results of this study indicate that there is a certain ratio of rotational angle for each segment, and suggest that differences in the ratio affect the local range of motion and muscle activities. Furthermore, with frequently repeated trunk rotation, the change in local range of motion may be one of the risk factors in low back pain.

Results of this study indicate that it is important for the LD and EO muscles to act coordinately during ipsilateral trunk rotation. Vera-Garcia et al. ${ }^{16)}$ reported that LD activation levels are similar to EO activation levels during thoracic rotation and that the LD is important for controlling upper trunk motions. In general, the LD is responsible for shoulder extension. In this study, it is likely that the LD produced thoracic rotation given that the subjects' arms were crossed over their chests. The results of this study indicate that the $\mathrm{IO}$ and TrA muscles act early during ipsilateral trunk rotation at lumbar levels. Urquhart et al. ${ }^{3)}$ reported that during ipsilateral trunk rotation, the IO and TrA muscle activity were significantly increased at lumbar levels when thoracic levels were immobilized. The results of this study indicated that during contralateral trunk rotation, the percentage of the MVC in the EO was significantly increased for all split ranges more than $40-50 \%$ and increased sharply for

Table 2. Each segment's maximal degree of rotation and the ratio to that observed for the upper thoracic (UTx) segment

\begin{tabular}{lllll}
\hline \multirow{2}{*}{ Segment } & \multicolumn{3}{c}{ Right } & \multicolumn{2}{c}{ Left } \\
\cline { 2 - 5 } & Degree & Ratio (\%) & Degree & Ratio (\%) \\
\hline UTx & $39 \pm 8$ & 100 & $39 \pm 9$ & 100 \\
MUTx & $35 \pm 7$ & $90 \pm 8$ & $35 \pm 6$ & $90 \pm 12$ \\
MLTx & $27 \pm 6$ & $70 \pm 7$ & $28 \pm 5$ & $72 \pm 7$ \\
LTx & $18 \pm 4$ & $48 \pm 7$ & $19 \pm 4$ & $49 \pm 6$ \\
ULx & $11 \pm 3$ & $30 \pm 6$ & $12 \pm 3$ & $30 \pm 6$ \\
LLx & $6 \pm 2$ & $16 \pm 5$ & $7 \pm 2$ & $19 \pm 7$ \\
\hline
\end{tabular}

All data are presented as mean \pm standard deviation.

UTx: upper thoracic segment (range from C7 to T3), MUTx: mid-upper thoracic segment (range from T3 to T6), MLTx: mid-lower thoracic segment (range from T6 to T9), LTx: lower thoracic segment (range from T9 to T12), ULx: upper lumbar segment (range from T12 to L3), LLx: lower lumbar segment (range from L3 to S1) 


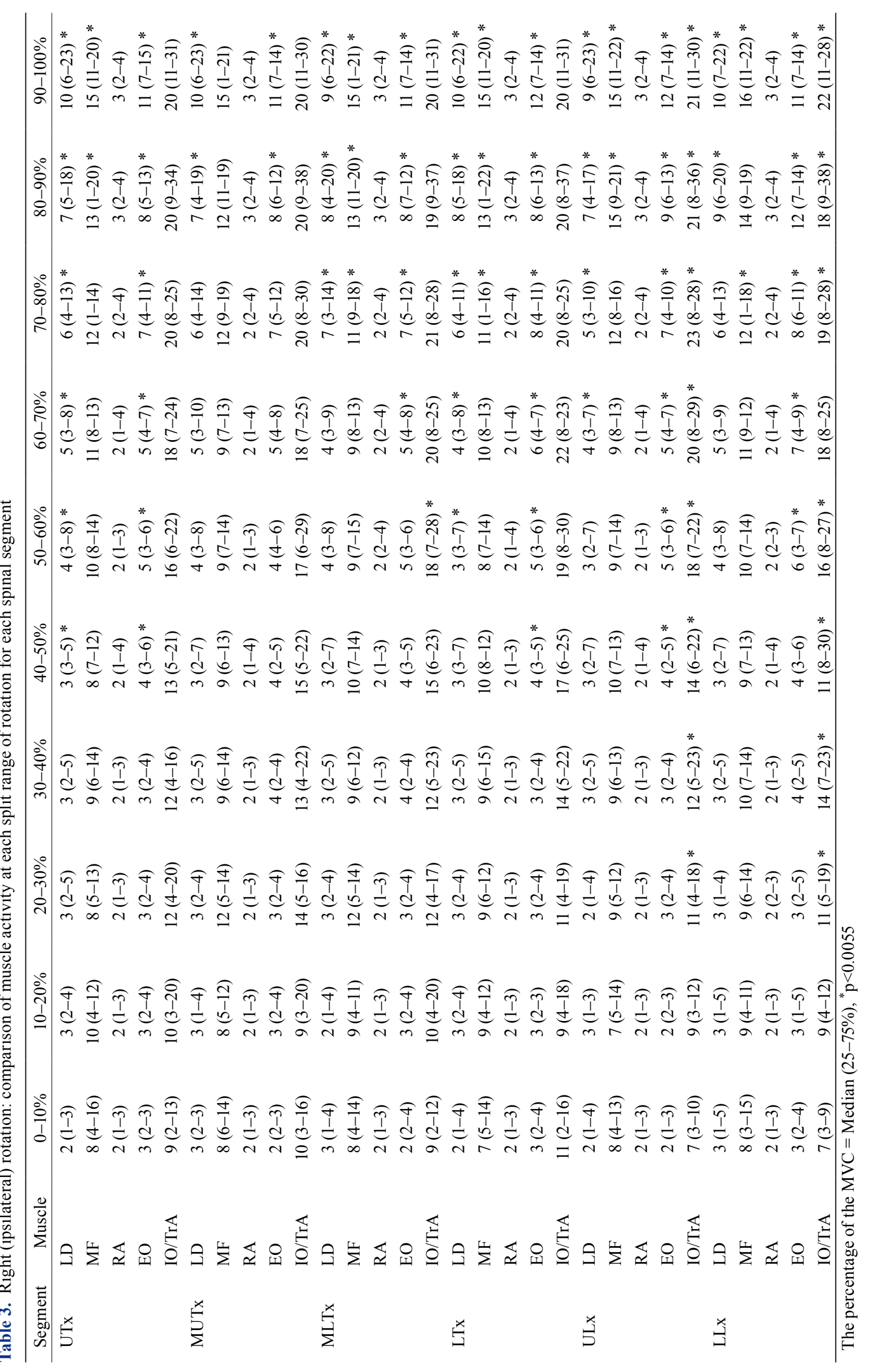




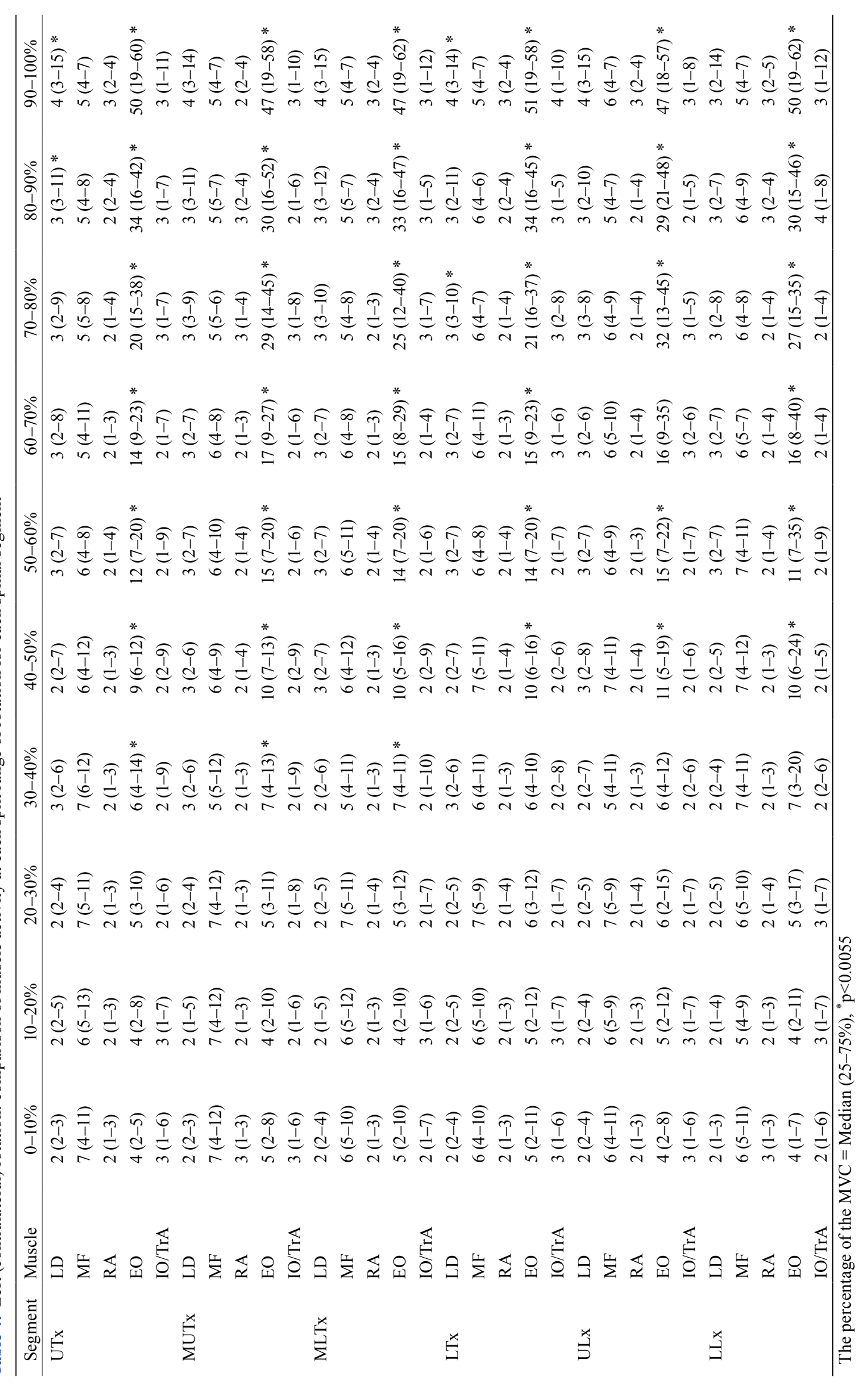


all split ranges more than $70-80 \%$. Torén ${ }^{5)}$ reported that the EO muscle activity was sharply increased during the final range of contralateral trunk rotation. Andersson et al. ${ }^{17)}$ reported that the percentage of the MVC in the IO was $58 \%$ and in the EO activity was $13 \%$ during ipsilateral trunk rotation; in addition, the IO was $16 \%$, the EO activity was $45 \%$ and the MF activity was $6 \%$ during contralateral trunk rotation. The results of this study are congruent with those previously reported.

There is little consensus regarding the function of the MF muscle. The method of manually resisted maximal voluntary contractions for the MF involves trunk extension and rotation toward the opposite side ${ }^{18)}$. On the other hand, axial rotation exerted by the MF is only minor on both sides ${ }^{19)}$. The results of this study suggest that the MF plays an important role in stabilization of the thoracolumbar region given that the muscle activity of the MF was greater than the other muscles from the starting position on both sides.

There are additional research questions to be addressed. First, trunk flexion, extension, and bending with rotation (coupling motion) were not evaluated $\left.{ }^{15}, 20\right)$. The relationship between muscle activities and these motions need to be examined. Second, the timing of abdominal and back muscle activity needs to be examined. Research into these areas will improve our understanding of trunk dysfunction.

\section{ACKNOWLEDGEMENT}

We are very grateful to the volunteers for their cooperation during this study.

\section{REFERENCES}

1) $\mathrm{Xu} Y$, Bach E, Orhede E: Work environment and low back pain: the influence of occupational activities. Occup Environ Med, 1997, 54: 741-745. [Medline] [CrossRef]

2) Rohrer MH, Santos-Eggimann B, Paccaud F, et al.: Epidemiologic study of low back pain in 1398 Swiss conscripts between 1985 and 1992. Eur Spine J, 1994, 3: 2-7. [Medline] [CrossRef]

3) Urquhart DM, Hodges PW: Differential activity of regions of transversus abdominis during trunk rotation. Eur Spine J, 2005, 14: 393-400. [Medline] [CrossRef]

4) Valencia FP, Munro RR: An electromyographic study of the lumbar multifidus in man. Electromyogr Clin Neurophysiol, 1985, 25: 205-221. [Medline]

5) Torén A: Muscle activity and range of motion during active trunk rotation in a sitting posture. Appl Ergon, 2001, 32: 583-591. [Medline] [CrossRef]

6) Hosea TM, Gatt CJ Jr: Back pain in golf. Clin Sports Med, 1996, 15: 37-53. [Medline]

7) Preuss RA, Popovic MR: Three-dimensional spine kinematics during multidirectional, target-directed trunk movement in sitting. J Electromyogr Kinesiol, 2010, 20: 823-832. [Medline] [CrossRef]

8) $\mathrm{Ng} \mathrm{JK}$, Kippers V, Richardson CA: Muscle fibre orientation of abdominal muscles and suggested surface EMG electrode positions. Electromyogr Clin Neurophysiol, 1998, 38: 51-58. [Medline]

9) Ishida H, Tajima S, Masuno R, et al.: Slow expiration reduces external oblique muscle activity during maximum trunk flexion. J Phys Ther Sci, 2014, 26: 1919-1921. [Medline] [CrossRef]

10) Waongenngarm $P$, Rajaratnam BS, Janwantanakul P: Perceived body discomfort and trunk muscle activity in three prolonged sitting postures. J Phys Ther Sci, 2015, 27: 2183-2187. [Medline] [CrossRef]

11) Vink P, Daanen HA, Verbout AJ: Specificity of surface-EMG on the intrinsic lumbar back muscles. Hum Mov Sci, 1989, 8: 67-78. [CrossRef]

12) Shin SJ, Kim TY, Yoo WG: Effects of various gait speeds on the latissimus dorsi and gluteus maximus muscles associated with the posterior oblique sling system. J Phys Ther Sci, 2013, 25: 1391-1392. [Medline] [CrossRef]

13) Sugaya T, Abe Y, Sakamoto M: Ultrasound evaluation of muscle thickness changes in the external oblique, internal oblique, and transversus abdominis muscles considering the influence of posture and muscle contraction. J Phys Ther Sci, 2014, 26: 1399-1402. [Medline] [CrossRef]

14) Gregersen GG, Lucas DB: An in vivo study of the axial rotation of the human thoracolumbar spine. J Bone Joint Surg Am, 1967, 49: 247-262. [Medline]

15) White AA 3rd, Panjabi MM: The basic kinematics of the human spine. A review of past and current knowledge. Spine, 1978, 3: 12-20. [Medline] [CrossRef]

16) Vera-Garcia FJ, Moreside JM, McGill SM: Abdominal muscle activation changes if the purpose is to control pelvis 
motion or thorax motion. J Electromyogr Kinesiol, 2011, 21: 893-903. [Medline] [CrossRef]

17) Andersson EA, Grundström H, Thorstensson A: Diverging intramuscular activity patterns in back and abdominal muscles during trunk rotation. Spine, 2002, 27: E152-E160. [Medline] [CrossRef]

18) Kendall FP, McCreary EK, Provance PG, et al.: Muscles Testing and Function with Posture and Pain, 5th ed. Philadelphia: Lippincott Williams \& Wilkins, 2005, pp 178-187.

19) Macintosh JE, Bogduk N: The biomechanics of the lumbar multifidus. Clin Biomech (Bristol, Avon), 1986, 1: $205-213$. [Medline] [CrossRef]

20) Moon OK, Kim SH, Lee SB, et al.: Thoracic coupled motions of Korean men in good health in their 20s. J Phys Ther Sci, 2014, 26: 87-91. [Medline] [CrossRef] 